\title{
Estrategias representacionales en deportistas: el control de las imágenes como herramienta facilitadora
}

\author{
JAIME DÍAZ OCEJO \\ ASPIRE Academy for Sports Excellence, Doha, Qatar \\ JUAN ANTONIO MORA MÉRIDA \\ Universidad de Málaga
}

LAS APROXIMACIONES CONTEMPORÁNEAS en relación al control perceptivo-cognitivo de los gestos técnicos en el deporte sugieren, básicamente, que los movimientos puedan ser planificados, ejecutados y almacenados en la memoria en forma de representaciones mentales y estén orientados a la anticipación del resultado (Schack y Tenenbaum, 2004). Ya en 1975, Nikolai Bernstein señaló que si consideramos el programa del acto del movimiento macroscópicamente como un todo, el único factor determinante que encontramos es la imagen del resultado. La imaginería, o la creación o recreación de experiencias sensoriales en la mente, supone la técnica psicológica más empleada por los deportistas de rendimiento y la más estudiada por la literatura científica en la Psicología del Deporte (Vealey, 2007). Así, la imaginería ha demostrado efectividad en el aprendizaje de habilidades deportivas, preparación para la competición, optimización del entrenamiento mental, para fomentar la motivación, el afrontamiento en situaciones de estrés y fortalecimiento de la auto-confianza (Nordin y Cumming, 2008; Hall, 2001).

En el presente trabajo, abordamos esta estrategia tan relevante en el entrenamiento de las representaciones mentales en el deporte haciendo referencia, en primer lugar, a una conceptualización adecuada y unívoca sobre la misma. En segundo lugar, se desarrollan las teorías explicativas más destacadas por la literatura científica, con el propósito de comprender los mecanismos que subyacen al funcionamiento de la imaginería. Seguidamente, en el tercer apartado, exponemos las funciones en el empleo de la imaginería y, finalmente, se analizan cuáles son las variables mediadoras relevantes con respecto a esta estrategia cognitiva, que aparece como base fundamental de la representación mental. 


\section{APROXIMACIÓN AL CONCEPTO DE IMAGINERÍA}

El término imaginería ha sido empleado de diversas formas, y con muy distintos matices, llevándolo en ocasiones a cierta confusión conceptual. Así, acepciones como ensayo simbólico, visualización, modelado, práctica encubierta, ensayo cognitivo, práctica imaginaria, sueños, entrenamiento viso-motor, entrenamiento ideo-motor e incluso entrenamiento de sofá se han referido, de forma equívoca, al mismo substrato cognitivo (Murphy y Jowdy, 1992).

Singer (1980) ya comentaba que algunos investigadores han definido la imaginería y práctica mental como términos análogos. Suinn (1993) aclaró a través de un ejemplo con el tenis, la diferencia conceptual entre la práctica mental y la imaginería, argumentando que, practicar mentalmente un servicio puede incluir pensar en el mismo, dialogar con uno mismo sobre cada paso utilizado, imaginarse sirviendo perfectamente o visualizando el golpeo adecuado, una vez ejecutada la acción. Por lo tanto, pedirle al deportista que realice la práctica mental no quiere decir que se haga referencia exclusivamente a la imaginería. En la línea apuntada por Suinn (1993), Murphy y Jowdy (1992) comentan que, aunque los psicólogos cognitivos han debatido acaloradamente sobre la naturaleza del proceso mental conocido como imaginería, parece haber consenso a la hora de afirmar que esta función cognitiva existe. Así, la imaginería se referiría a un proceso mental mientras que la práctica mental sería un término descriptivo para una técnica en particular, utilizada por deportistas y muchos otros individuos (Murphy y Jowdy , 1992).

En el contexto de este capítulo, como es el deporte, sería pertinente considerar al fenómeno de la imaginación como un proceso que integraría a varios puntos de codificación, más allá de una mera referencia de almacenamiento verbal. Así, el deportista de élite puede interpretar un problema táctico a través de la representación que hace del mismo, y decodificar la información entrante a la vez que accede a la memoria a largo plazo para generar soluciones hacia la mejora del rendimiento (McPherson y Vickers, 2004). Del mismo modo, cabría diferenciar la imaginería en el deporte de las imágenes mentales que se manifiestan, por ejemplo, en el pensamiento infantil (Mora y Díaz Ocejo, 2008; Díaz Ocejo, 2006). En efecto, tanto Piaget e Inhelder (1971) como Bruner (1960), parecen aceptar que existe una rigidez de la imagen mental en las etapas tempranas de la infancia (representaciones figurativas), que van modificándose hasta convertirse en representaciones simbólicas (que permiten representar mentalmente objetos en su ausencia), más propios de etapas más maduras y características de los adultos. 


\section{TEORÍAS EXPLICATIVAS DE LA IMAGINERÍA}

La literatura científica refleja diversas conceptualizaciones acerca de su concepción teórica. Algunos autores como Suinn (1993), han destacado la Teoría Psiconeuromuscular, la Teoría del Aprendizaje, la Teoría de la Atención y la Teoría Bioinformacional. Otros como Gould y Damarjian (1996), han considerado relevantes la Teoría Psiconeuromuscular, la Teoría del Aprendizaje Simbólico y las teorías basadas en los procesos motores y de información como son la Teoría de Lang $(1977,1979)$ y la Teoría del Triple Código de Ashen (1984). Hall (2001), por su parte, destaca la Teoría del Aprendizaje Simbólico, la Teoría Psiconeuromuscular, la Teoría Bioinformacional y la Teoría del Código Dual. Otras revisiones como la realizada por Sánchez y Lejeune (1999), han hecho referencia a la Teoría Psiconeuromuscular, la Teoría del Aprendizaje Simbólico, la Teoría Bioinformacional, la Teoría del Triple Código y otros enfoques teóricos como son las teorías de la Regulación Atencional, Condicionamiento Interno y Teoría de la Auto-eficacia. No obstante, parece evidente que estas teorías explicativas pueden agruparse en dos grandes bloques; aquellas que han utilizado un punto de vista fisiológico en sus explicaciones, y las que se han posicionado desde una perspectiva cognitiva.

\section{II.1. LAS TEORÍAs PSiconeuRomusCULARES}

Estas teorías se basan en el presupuesto de que existe una equivalencia funcional entre imaginar y realizar una acción, tal como se procesa en el sistema nervioso. De ese modo, al imaginar una determinada acción, se desencadenan impulsos nerviosos en las fibras motoras que estarían implicadas en el movimiento real, generándose unos patrones neuromusculares muy inferiores a los que se producen en la práctica física. Por lo tanto, la naturaleza de la estimulación neuromuscular en la imaginería sería muy similar a la que se produce en las ejecuciones físicas reales. Es decir, el mecanismo neuronal sería similar en ambos procesos, el de la imaginería motora y el de la ejecución motora (Smith, Wright, Allsopp y Westhead, 2007; Ramsey, Cumming y Edwards, 2008).

Otra teoría que se fundamenta en las conexiones existentes entre el cerebro y las terminaciones nerviosas en los músculos, es la aportada por Eccles (1972). En su Hipótesis del Aprendizaje Neuronal, este autor defiende que el proceso bioquímico de transmisión de impulsos nerviosos en una fibra, se optimiza cuantos más impulsos se transmitan a esa fibra. Lo que significaría un aprendizaje o entrenamiento de la conducción neuromuscular, incluso en la práctica de la imaginación. Similarmente, la Hipótesis del Feedback Cinestésico de Corbin (1972), apunta a que, cuando se imagina una acción, los impulsos que se desencadenan en las fibras motoras proporcionan una retroalimentación 
que favorece las correcciones oportunas para que el sujeto mejore su ejecución en el movimiento real.

Más orientada, quizá, a la neurofisiología, McKay (1981) propuso su Teoría de la Activación Nodular. En esta Teoría existiría una conexión funcional entre los nódulos que son responsables en los procesos de imaginación, y los nódulos que propician la ejecución del movimiento. Así, la activación de los nódulos mentales que se produce cuando se imagina, conllevaría un efecto preparador en los nódulos responsables del movimiento muscular.

Algunos estudios como el de Ranganathan, Siemionow, Liu, Sahgal y Yue (2004), han sido representativos de las orientaciones psiconeuromusculares. Estos autores examinaron los efectos que la imaginería podría tener en dos tareas de fuerza, en comparación con la ausencia de esta práctica. De ese modo, los sujetos $(n=30)$ fueron distribuidos en tres condiciones distintas; en la primera

(A)

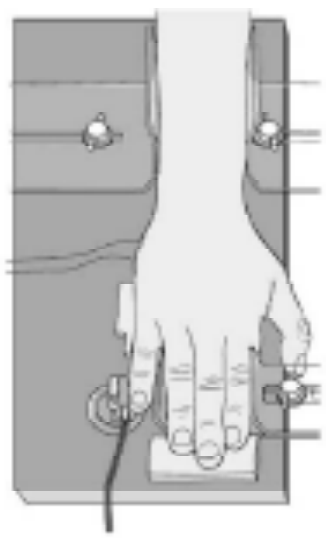

(B)

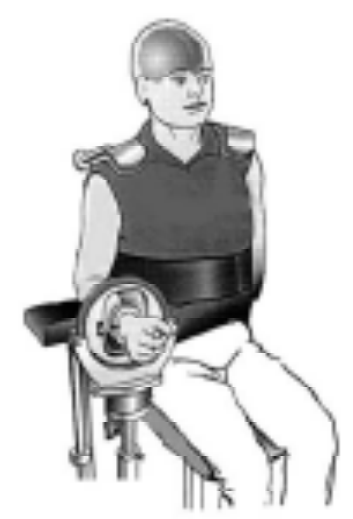

(C)

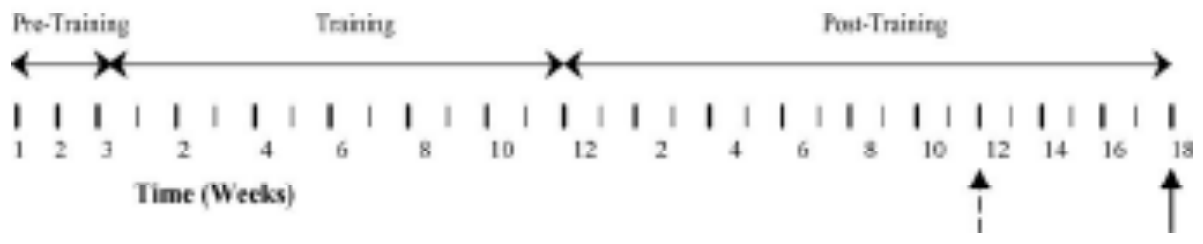

Figura 1. Representación de la tarea de fuerza con el dedo (A), y de la tarea de fuerza de flexión del codo (B). La línea horizontal muestra el tiempo de la experiencia (en semanas), incluyendo los períodos de prestest, entrenamiento y postest. La línea vertical discontinua representa el final del entrenamiento para la condición de la tarea de fuerza con el codo y, la línea vertical continua el final del entrenamiento de la tarea de fuerza con el dedo meñique.

Tomado de Ranganathan, Siemionow, Liu, Sahgal y Yue, (2004, p. 946). 
los sujetos fueron instruidos para que entrenaran mentalmente la contracción muscular en una tarea de abducción del dedo meñique, la segunda condición fue instruida también para que entrenara mentalmente la contracción muscular de la flexión del codo, a la tercera no le fue asignada ninguna instrucción aunque tomaron parte en las medidas registradas y, el cuarto grupo fue instruido para que entrenara la fuerza máxima en la abducción del dedo meñique. El entrenamiento duró 12 semanas, 15 minutos cada día durante cinco días a la semana.

Los resultados pusieron de manifiesto que el grupo que había entrenado mentalmente la abducción del dedo meñique, mejoró en un 35\% los registros de fuerza. Igualmente, el grupo que empleó la imaginería para el entrenamiento de la fuerza en la prueba de flexión del codo, mejoró sus resultados en un $13.5 \%$. La condición que había entrenado físicamente la abducción de fuerza maximal con el brazo aumentó su rendimiento en un 53\% y, el grupo control (al que no había sido asignado ninguna instrucción) no mostró mejoras significativas. Los autores concluyeron, en base a los datos obtenidos y desde una perspectiva de la aplicación clínica que "nuestros resultados demuestran que la mente ejerce un poder destacable sobre el cuerpo y sus músculos" (Ranganathan, Siemionow, Liu, Sahgal y Yue, 2004, p. 955). Aunque, tal y como mostraron los porcentajes, la condición asignada a la práctica física obtuvo los mejores resultados.

No obstante, las teorías con enfoque psiconeuromuscular, aunque tienen cierta fiabilidad científica y añaden algunas afirmaciones interesantes para el empleo de la imaginería en el ámbito del ejercicio físico, han mostrado, por otra parte, importantes carencias metodológicas. Igualmente, autores como Johnson (1982) o Kohl y Roenker $(1980,1983)$, desde el punto de vista de la psicología experimental, no han obtenido evidencias de que los efectos de la práctica mental y, por ende, de la imaginería, puedan ser explicados desde la actividad muscular.

\section{II.2. LAS TEORÍAS DEL APRENDIZAJE SIMBÓLICO}

Las hipótesis relacionadas con el aprendizaje simbólico, también denominadas teorías simbólico-perceptuales (Denis, 1985), se fundamentan en los procesos mentales para defender que la codificación simbólica de las imágenes representadas en la mente, optimizan la organización perceptiva y estructuración del gesto a realizar, a nivel cognitivo. Esta aproximación cognitiva a la explicación de este fenómeno, mantiene que las acciones son simbólicamente codificadas a modo de huella mental (Vealey y Walter, 1993). Así, la imaginería reforzaría esa huella mental posibilitando que las acciones sean más familiares y posiblemente más automatizadas. De acuerdo con esta hipótesis, las habilidades que son más cognitivas por su naturaleza (como trazar un laberinto sobre un papel), son mejor codificadas que las habilidades motoras puras (Hall, 2001). 
Sackett $(1934,1935)$ fue el primero en proponer la Teoría del Aprendizaje Simbólico, a través de las experiencias obtenidas con el empleo de laberintos de lápiz y papel, que tenían un alto componente cognitivo. Otros estudios apoyaron las conclusiones aportadas por Sackett $(1934,1935)$ como el trabajo de Perry (1939), quien empleó cinco tareas diferentes para examinar los efectos de la imaginería, o Morrisett (1956), quien encontró que en una tarea de alto componente simbólico existían diferencias entre los grupos de práctica física y de imaginería, y que, además, habían diferencias entre estos dos grupos y la condición control.

Wrisberg y Ragsdale (1979) por su parte, encontraron que la imaginería conllevaba efectos superiores en un test de alto componente simbólico, que en una tarea de equilibrio (bajo componente simbólico). No obstante, aunque la mayoría de las investigaciones mostraron resultados favorables al igual que los presentados por Sackett $(1934,1935)$, Ryan y Simons (1983) hallaron que, en una tarea predominantemente motora (equilibrio en estabilómetro), no existían diferencias entre las condiciones de imaginería y control. Por otro lado, en la tarea de alto contenido simbólico (trazar un laberinto), ambos grupos, el de entrenamiento en imaginería y físico, mejoraron significativamente más que la condición control. Similarmente, Rawlings, Rawlings, Chen y Yilk (1972) no encontraron diferencias significativas entre los grupos de imaginería y práctica física en una tarea de alto componente motor, o Smyth (1975) quien concluyó que la imaginería no era efectiva ni en tareas de componente simbólico ni en tareas de componente motor.

Por su parte, Sage (1977) presenta su Hipótesis Simbólico Perceptual a través de la cual explica que la práctica en imaginería de una secuencia de movimientos, fomenta unos insights (algo parecido a la introspección consciente) perceptuales del patrón de movimientos. Este proceso desencadenaría una mejora en la ejecución del movimiento, fundamentalmente a través de una organización perceptiva del gesto a ejecutar.

Otro autor que ha presentado su teoría, en consonancia con la hipótesis declarada por Sackett (1934, 1935), ha sido Mackay (1981). Su Teoría de la Adquisición de Habilidades Seriales "involucra una jerarquía de nódulos interconectados que son responsables de controlar una secuencia organizada de acciones" (Hall, 2001, p. 538). De ese modo, la infraestructura de esa jerarquía se asegura de que los componentes de la habilidad se ejecuten de acuerdo con la secuencia fijada. En lo más alto de la jerarquía, se situaría el nódulo que representa el comportamiento completo, y los nódulos que se situarían más abajo son los responsables de los distintos músculos. Así, la activación normalmente se inicia desde arriba, con el fin de controlar el movimiento. Mackay (1981) ha obtenido evidencias favorables que sustentan su Teoría en una tarea de lenguaje, aunque Annet (1988), no ha podido corroborar esos resultados en otras tareas seriales. 
La teoría del aprendizaje simbólico deja algunas cuestiones sin responder: Explica cómo los practicantes noveles pueden beneficiarse con la práctica de la imaginería, esta práctica fortalece las huellas mentales de la nueva habilidad que se aprende, sin embargo, la teoría no revela cómo el rendimiento mejora en practicantes experimentados que ya han optimizado las habilidades necesarias. Aún más, es difícil determinar el tamaño del componente cognitivo de una tarea motora (Hall, 2001). Por lo tanto, estos argumentos hacen que la orientación del aprendizaje simbólico, al igual que ocurría con la aproximación psiconeuromuscular, sea tenida en cuenta como una hipótesis que necesita ser revisada y actualizada, sobre todo en consonancia con modelos más recientes como los ecológicos de imaginería o de control motor.

\section{II.3. La Teoría Bioinformacional o de Procesamiento de la Información DE $\operatorname{LANG}(1977,1979)$}

Este modelo, originalmente desarrollado para explicar algunas patologías clínicas relacionadas con las fobias y la ansiedad, se orienta desde una perspectiva de imaginería de procesamiento de la información (Lang, 1977, 1979). El modelo se fundamenta en que una imagen es un conjunto (set) funcionalmente organizado y finito de proposiciones almacenadas en el cerebro. Así, la imagen se describiría en base a dos supuestos o proposiciones, las proposiciones de estímulos y las proposiciones de respuesta, con lo que la imaginería implicaría la activación de ambos códigos proposicionales que serían almacenados en la memoria a largo plazo (Hernández Mendo, 2002). Las proposiciones de estímulo implican afirmaciones que describen el contenido de la escena que ha de ser imaginada, mientras que las proposiciones de respuesta son las afirmaciones que describen la respuesta del que imagina esa escena. Es decir, las primeras aludirían a elementos descriptores acerca del estímulo (por ejemplo, el peso de la raqueta en el tenis), mientras que las segundas harían referencia a afirmaciones relativas al comportamiento, como podría ser la tensión muscular creada para agarrar la raqueta (estas proposiciones de respuesta son medibles a través del uso del EMG).

Así, el comportamiento representado implicaría la evaluación de proposiciones de estímulo con su correspondiente reacción de las proposiciones de respuesta. Por lo que la imagen no sería solamente un estímulo en la mente del que imagina, sino más bien, una interpretación realizada a través de las proposiciones de respuesta, aunque estas deben ser activadas a partir de las proposiciones de estímulo. Según Lang, Melamed y Hart (1970), al ser las proposiciones de respuesta más modificables y representar cómo un individuo podría reaccionar en un contexto real, éstas pueden tener un impacto mayor en el comportamiento subsiguiente. Es decir, un jabalinista podría incluir en sus 
sesiones de imaginería síntomas físicos sobre activación o ansiedad, mientras que ejecuta los lanzamientos. Esto podría facilitar su rendimiento en la realidad, en comparación con la práctica en imaginería sin incluir esos síntomas (estímulos). Del mismo modo, las instrucciones para la práctica de la imaginería con las proposiciones de respuesta, deberían facilitar bastantes más respuestas de tipo fisiológico que las instrucciones relativas a las proposiciones de estímulo (Budney, Murphy y Woolfolk, 1994). Esta consideración indicaría que "es la interacción entre el modo de entrenamiento y la estructura proposicional de la representación de la imaginería lo que resulta crucial" (Carroll, Marzilier y Merian, 1982, p. 76).

Otros autores como Hale (1994) han señalado que las diferencias entre ambas proposiciones son funcionalmente similares a las encontradas entre la imaginería interna y externa. Evidencia que parte de los trabajos realizados por Hale (1982) y Harris y Robinson (1986), con respecto a que la imaginería empleada desde una perspectiva interna provoca mayor actividad en el EMG que la producida por la imaginería externa.

Por su parte y en la línea de la perspectiva de la Teoría Bioinformacional, Holmes y Collins (2001) presentan su modelo de equivalencia funcional denominado PETTLEP (physical, environmental, task, timing, learning, emotional y perspective).

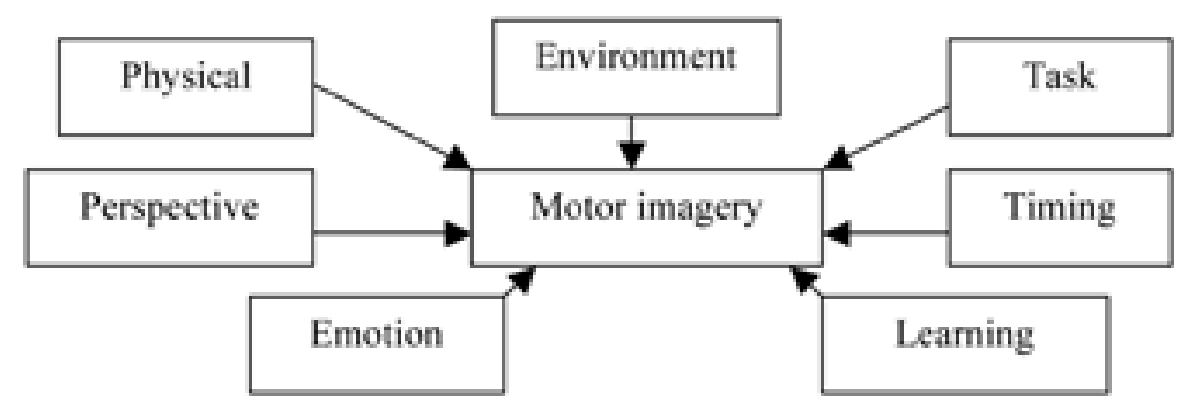

Figura 2. Representación del Modelo PETTLEP con las interacciones entre la imaginería motora y los siete elementos que interactúan con ella. Adaptado de Holmes y Collins (2001, p. 70).

Este modelo, que incluye elementos físicos, del entorno, tarea, aprendizaje, emocionales y de perspectiva, se apoya en la afirmación de que el cerebro utiliza la memoria en forma de representación central, a la que tienen acceso tanto la preparación y ejecución física como la imaginería motora que se asocia a ambas. Por lo tanto, estos autores proponen con este Modelo que se ha hecho poco desde la psicología del deporte para comprender la relación 
entre la imagen motora y el movimiento que representa y la forma en que su relación puede ser explotada para ser optimizada, mientras que la efectividad de la imaginería motora para mejorar el rendimiento es claramente multifactorial, muchos de estos factores parecen tener enlaces con la tarea física cuando la imaginería tiene éxito (Holmes y Collins, 2001). Teniendo en cuenta estas consideraciones, los autores se basan en los siete elementos que conforman su Modelo, como requisitos para que los psicólogos del deporte monitoricen esa equivalencia entre la tarea física y la mejora de la práctica en imaginería. No obstante, estos autores son conscientes de que este Modelo se encuentra lejos de estar completo y animan a los investigadores a que orienten sus trabajos en esta dirección.

Igualmente, y aunque la orientación presentada por la Teoría Bioinformacional o de Procesamiento de la Información de Lang $(1977,1979)$ parece ser una mejora con respecto al aprendizaje simbólico y la teoría psiconeuromuscular; sin embargo, se basa en la psicofisiología y explica relativamente poco sobre las funciones motivacionales que sirve la imaginería. Tampoco aborda el papel de la imaginería en acción integradora con otras formas de procesamiento de la información tales como el lenguaje (Hall, 2001).

Como se puede apreciar a partir de estas indicaciones, cada orientación desde la que se enfoca un criterio para explicar a la imaginería aporta algunas consideraciones novedosas, si bien todas parecen carecer u obviar otros factores no menos relevantes.

\section{II.4. La Teoría DEL TRIPLE Código de Ashen (1984), (ImaGe-SomatiC- RESPONSE-MEANING, ISM).}

Este modelo de imaginería también destaca el papel que los procesos psicofisiológicos tienen durante la representación mental. Para Ashen (1984), existiría un paso más hacia delante necesario para describir el fenómeno de la imaginería y que, por lo tanto, debe ser incluido como Teoría relativa a la explicación de la misma. El autor se refiere a la importancia que el significado de la imagen tiene para el individuo, factor que ha sido ignorado por las otras teorías explicativas. En efecto, de acuerdo con esta propuesta, se presentan tres elementos que deben componer el proceso de la imaginería. En primer lugar la imagen, a la que Ashen (1984, p. 34) se refiere como "una sensación de activación controlada. Posee todos los atributos de la sensación pero es interna al mismo tiempo. Representa el mundo exterior y sus objetos con un grado de realismo sensorial que nos permite interactuar con la imagen como si estuviéramos interactuando con el mundo real". La segunda parte es la respuesta somática, que al igual que otros autores (Lang, 1977, 1979; Sheik y Kunzendorf, 1984) han puesto de manifiesto cuando se lleva a cabo la imaginería, se 
producen cambios en el cuerpo a nivel psicofisiológico. Finalmente, el tercer componente de esta Teoría (aspecto novedoso de la misma) es la significación que la imagen tendría para el individuo.

Es decir, de acuerdo con Ashen (1984), cada imagen conlleva una significación para el individuo que la imagina, por lo que el mismo conjunto de instrucciones para la imaginación nunca producirá la misma experiencia para dos personas distintas. Así pues, cuando se construyen imágenes mentales, resulta siempre útil evaluar las experiencias anteriores del individuo para adecuar la visualización al significado que la imagen puede tener para el mismo (Chiaie, D’Ippolito, Gramaccioni, Polani, Reda, Régine, Robazza y Sacco, 2004).

Murphy y Martin (2002) han sugerido recientemente acerca de la práctica en imaginería, que las investigaciones al respecto han ofrecido poco para comprender los procesos cognitivos que subyacen al empleo de esta estrategia. Estos autores proponen tres niveles desde los que la imaginería debería practicarse, como son la naturaleza de la misma, las posibilidades de su empleo en el contexto del deporte y la significación que esta experiencia tiene en los deportistas.

\section{II.5. OTRAS PERSPECTIVAS EXPLICATIVAS DE LA IMAGINERÍA}

Tal y como se ha presentado, las distintas perspectivas desde las que se ha abordado el concepto de la imaginería, han supuesto de algún modo un progreso o continuo para comprenderla mejor. En ese sentido, se han establecido las diferentes teorías o modelos que van desde las que consideran al proceso de imaginar como un proceso cercano a la fisiología del cuerpo humano (teorías psiconeuromusculares), pasando por las concepciones más ligadas al procesamiento de la información (Lang, 1977, 1979), hasta las que consideran al individuo como elemento fundamental subjetivo, que interactúa con el entorno y proporciona su propia significación al objeto que debe ser imaginado. $\mathrm{Al}$ margen de las teorías que tienen como fin explicar los procesos que tienen lugar durante la práctica de la imaginería, otras perspectivas han mostrado su punto de vista a este respecto.

Annett $(1988,1994)$ ha propuesto su Modelo del Código Dual (Actionlanguage-imagination, $A L I$ ) en el que, a partir del campo motor, existirían dos vías a través de las cuales el deportista puede adquirir información acerca de una habilidad; una sería la demostración y otra la instrucción verbal. Estas, a su vez, se basan en dos canales de codificación independientes (el canal motor y el canal verbal). El canal motor sería el responsable de codificar la acción humana, mientras que el canal verbal, el que codificaría las acciones del habla y el lenguaje (incluyendo el lenguaje escrito). De ese modo, se establece un puente entre los dos canales, denominado puente de la acción-lenguaje. Partien- 
do de las aportaciones presentadas en la revisión de Paivio (1986), apoyando la conexión existente entre la memoria y el aprendizaje, las experiencias de la Teoría del Código Dual de Annett $(1988,1994)$ propone que la codificación de la información en ambos sistemas de acción y lenguaje, debería producir un mejor aprendizaje que la utilización de una de ellas únicamente.

Por otra parte, la Teoría de la Activación en la imaginería, se posiciona desde una orientación en la que esta práctica fomenta establecer un nivel de excitación, o activación psicológica óptimo que facilite la ejecución. Similarmente, es sabido que un estado adecuado de vigilia es también necesario para fomentar la ejecución requerida. Así, de acuerdo con Schmidt (1982, p. 520) "el ejecutante está preparado para la acción, manteniendo el nivel de excitación y generalmente aportando preparación para un buen resultado", por lo que el nivel de activación del deportista cobra especial relevancia en esta Teoría. Para la relación activación-ejecución, los procesos atencionales jugarían un papel importante ya que, un estado óptimo de vigilia permite que la práctica en imaginería optimice la concentración durante las ejecuciones deportivas.

Por lo tanto, ambas teorías (Teoría de la Activación y Teoría de la Regulación Atencional), aportan una explicación de los procesos que involucran la práctica de la imaginería en relación a la excitación, su influencia en la atención e incluso con las respuestas emocionales. Sin embargo, estas suposiciones no han tenido repercusiones consistentes en la literatura científica.

Otros enfoques teóricos relacionados con la imaginería son, por un lado, la Teoría del Condicionamiento Interno (basada en el principio de los condicionamientos encubiertos de Cautela y Sambperil, 1989) en la que las respuestas internas y externas tienen efectos similares en el cuerpo, partiendo de los mismos principios de aprendizaje y condicionamiento. Por lo que, el entrenamiento y práctica de una determinada imagen mental junto con las retroalimentaciones acordes con la ejecución deseada, conllevarían a la consiguiente mejora de la ejecución. Por otro lado, la facilitación que la imaginería tiene sobre las expectativas del deportista, de acuerdo con la Teoría de la Auto-Eficacia de Bandura (1977). Esta perspectiva deberá tenerse en cuenta, a partir de la información que el deportista extrae de la demostración, que, de ser la adecuada, optimizaría tanto el proceso de aprendizaje como el rendimiento.

Tal y como se ha podido constatar en lo revisado acerca de las teorías explicativas sobre el fenómeno de la imaginería, aunque han sido diversas las aproximaciones a la misma (fundamentalmente las derivadas de las perspectivas psicofisiológicas y del procesamiento de la información), todas pueden tener en común que manifiestan parcialmente supuestos evidentes al respecto, si bien ninguna ofrece una postura concluyente para la comprensión de la imaginería. Similarmente, y como se ha mostrado en algunas investigaciones desarrolladas en este ámbito, los resultados obtenidos en tareas motoras o en 
el deporte en general tampoco son concluyentes, al igual que ocurre con los ofrecidos a nivel genérico.

\section{El EMPLEO DE LA IMAGINERÍA EN EL ÁMBITO DE LA ACTIVIDAD FÍSICA Y EL DEPORTE}

Según autores como Vealey (2007) o Nordin y Cumming (2008), la influencia que el uso de la imaginería tiene en el contexto del deporte, puede ser comprendida a través del marco analítico propuesto por Paivio (1985). Este autor sugirió que esta práctica presta dos servicios que actúan a nivel específico, o a nivel general. Por un lado, la función cognitiva del ensayo de las habilidades propias de la actividad a desarrollar, denominada imaginería cognitivoespecífica (como por ejemplo imaginarse ejecutando un saque en tenis). Por otro, las estrategias referidas a la preparación de la competición, denominada imaginería cognitivo-general (como puede ser examinar visualmente el terreno donde se desarrollará una carrera de campo a través). Paivio (1985) describió su marco conceptual con el objetivo de fomentar el aprendizaje y rendimiento, no sólo a través de la función cognitiva, sino también incluyendo las funciones motivacionales. Así, este autor distingue entre la función motivacional a nivel específico, que incluiría imaginar la meta en sí y las actividades necesarias para alcanzarlas (como puede ser verse en lo más alto del podio), y la función motivacional a nivel general, que se referiría a imaginar estados generales de activación fisiológica y emocionales, como pueden ser las técnicas designadas para alcanzar estados de relajación o, por el contrario, fomentar un aumento del nivel de activación.

\begin{tabular}{|c|c|c|}
\hline & Motivacional & Cognitivo \\
\hline Específico & $\begin{array}{l}\text { Respuestas orientadas a la meta } \\
\text { (ejemplo, imaginarse a uno mismo } \\
\text { ganando una competición y recibiendo } \\
\text { una medalla) }\end{array}$ & $\begin{array}{l}\text { Habilidades (ejemplo, imaginarse } \\
\text { ejecutando bien sobre la barra de } \\
\text { equilibrios) }\end{array}$ \\
\hline General & $\begin{array}{l}\text { Activación (ejemplo, incluir la re- } \\
\text { lajación para imaginarse un lugar } \\
\text { tranquilo) }\end{array}$ & $\begin{array}{l}\text { Estrategia (ejemplo, imaginarse llevar } \\
\text { a cabo una estrategia para ganar una } \\
\text { competición) }\end{array}$ \\
\hline
\end{tabular}

Tabla 1. Funciones cognitivas y motivacionales de la imaginería propuestas por Paivio (1985). Traducido de Weinberg y Gould (2003, p. 287). 
En palabras de Weinberg, Butt, Knight, Burke y Jackson (2003, p. 27) acerca de esta conexión cognitivo-motivacional, "ambas funciones cognitivas operan en dos niveles: general y específico. Esta delineación de la función de la imaginería resulta en un modelo octogonal de $2 \times 2$ con el contraste cognitivo-motivacional como una dimensión y, el contraste general específico, como otra".

Posteriormente, y con el objetivo de completar esta conceptualización analítica, Hall, Mack, Paivio y Hausenblas (1998) identificaron dos componentes específicos de la imaginería motivacional general; por un lado, la imaginería motivacional general de activación (motivational general-arousal) que se asocia al estrés y el nivel de activación y, por otro, la imaginería motivacional general de éxito o maestría (motivational general-mastery), que implicaría el estado de control, auto-confianza y dureza mental del deportista. En la mayoría de los estudios sobre la imaginería se ha examinado la imaginería cognitivo-específica, que tal y como se ha apuntado, hace referencia a las actividades orientadas a la mejora del rendimiento.

\section{VARIABLES MEDIADORAS EN LA EFECTIVIDAD DE LA IMAGINERÍA}

En relación a la perspectiva interna (cuando la persona se imagina la situación deportiva desde dentro, tal cual ella la percibe) o externa (cuando se imagina desde fuera de sí mismo, tal y como lo haría si se viese en televisión) del empleo de la imaginería como herramienta facilitadora para las representaciones mentales de los deportistas, no se han determinado resultados concluyentes. Meyers, Cooke, Cullen y Liles (1979) no encontraron diferencias significativas en el tipo de perspectiva utilizada por jugadores de raquetball (deporte similar al squash), Epstein (1980) tampoco halló diferencias significativas a favor de ninguna de las dos perspectivas en un deporte de precisión como el lanzamiento de dardos o Mumford y Hall (1985), revelaron que no existían diferencias significativas en la perspectiva de uso de la imaginería en patinadores sobre hielo, en relación a la imaginería visual interna, visual externa y la cinestésica interna. Otros trabajos como el de Cumming y Ste-Marie (2001) con patinadores sobre hielo o Hardy y Callow (1999) con deportistas de deportes individuales, encontraron resultados que pusieron de manifiesto que la perspectiva externa fue la más empleada. Sin embargo, más recientemente Callow y Hardy (2004) han obtenido resultados inconsistentes, esta vez con una población de estudiantes de educación física en una tarea motora de esquema corporal (coordinación de movimientos del brazo y mano en determinadas partes del cuerpo). Por lo tanto, no existe consenso científico acerca de qué perspectiva es la más eficaz para el deporte. 
En relación a la controlabilidad y viveza de las imágenes, uno de los primeros trabajos en medir ambas, en un deporte individual como la gimnasia, fue el llevado a cabo por Start y Richardson (1964). Estos autores, no encontraron una correlación entre la habilidad de los gimnastas en estas variables de la imaginería, en relación con el aprendizaje y rendimiento de ejecuciones en ese deporte. En consonancia con esos resultados, en el estudio de Mahoney y Avener (1977) tampoco se encontraron evidencias entre el control de la imaginería en relación al rendimiento en gimnasia. Similarmente, Epstein (1980), no halló diferencias entre el empleo de esta estrategia cognitiva y la precisión en el lanzamiento de dardos. No obstante, otras evidencias se han mostrado inconsistentes en lo relativo a la habilidad en el uso de la imaginación, en comparación con las anteriores. Así, Goss, Hall, Buckolz y Fishburne (1986), concluyeron en su investigación a través de otra tarea motora, que los sujetos con mayores puntuaciones (medidas a través del Movement Imagery Questionnaire MIQ de Hall y Pongrac, 1983) de habilidad en imaginería tanto visual como cinestésica, aprendían y ejecutaban la tarea mejor que los sujetos con puntuaciones inferiores en la escala MIQ. Más interesante aún, Hall, Buckolz y Fishburne (1989), realizaron un estudio parecido al de los autores anteriores en el que, en esta ocasión, se medía únicamente la capacidad de los sujetos para recordar ciertos patrones de movimiento. Aunque no hubo diferencias significativas entre los grupos experimental y control en cuanto a rendimiento, los que imaginaban mejor recordaban con mayor precisión el patrón del movimiento a revivir. Por lo tanto, parece que existe una relación entre la capacidad de controlar y vivir las imágenes y un mejor rendimiento en el deporte.

La dificultad de la tarea es otra variable relevante a tener en consideración, a la hora de comprender cómo los deportistas utilizan la imaginería. Sin entrar en el análisis de la significación de la complejidad motora (duración o tiempo de movimiento, la fuerza requerida, la dirección, la extensión, la precisión, y el número de segmentos corporales involucrados), de acuerdo con Hall, Schmidt, Durand y Buckolz (1994), el aprendizaje y rendimiento de las tareas motoras más sencillas también supone más facilidad para imaginarlas y, por lo tanto, se recuerdan con más facilidad. Así, estos autores sugieren que las habilidades complejas, al ser más difíciles de imaginar, puedan incorporar para su mejor identificación en la memoria un elemento verbal para facilitar su aprendizaje y rendimiento.

Finalmente, en relación a los efectos de las imágenes positivas, en comparación con las negativas, y aunque parece demostrado que el empleo de la imaginería positiva puede ser más beneficiosa para la práctica deportiva, esto no significa que hubiera que eliminar las imágenes negativas en ciertos momentos de los entrenamientos (Hall, Schmidt, Durand y Buckolz, 1994). De acuerdo con esto, en determinadas especialidades donde la ejecución técnica es 
fundamental (como ocurre en el caso de muchos deportes individuales), sería interesante que los deportistas pudieran utilizar la imagen negativa a efectos de reconocer, congelar y corregir acciones técnicas ejecutadas incorrectamente. Sin embargo, un aspecto que todavía parece no estar claro sería el modo en que las imágenes negativas pueden interferir de forma negativa en el rendimiento deportivo.

\section{CONSIDERACIONES FINALES}

A raíz de lo analizado en este capítulo, parece que la práctica en imaginería ejerce una influencia positiva en el rendimiento deportivo y supone una herramienta facilitadora para la representación mental del deportista. No obstante y si nos atenemos a las evidencia sustentadas en la ciencia, por un lado, las teorías explicativas de este fenómeno no parecen definitivas a la hora de aclarar todos los procesos que abarca el empleo de esta estrategia cognitiva, aunque todas aportan conclusiones parciales interesantes y fiables. Por otro lado, la mayoría de las investigaciones llevadas a cabo en este ámbito, se han practicado desde la orientación de la imaginería cognitivo-específica, no considerando en tanta medida otras perspectivas presentadas por Paivio (1986), como son la imaginería cognitivo-general o las relacionadas con los procesos motivacionales de los deportistas. Probablemente, este hecho sea debido a que la percepción de la efectividad de la representación en la imaginación cognitivo-específica sea más efectiva para la ejecución de los gestos deportivos (Nordin y Cumming, 2008).

Similarmente, otro marco experimental desarrollado ampliamente, como el que abarca la diferencia del empleo de esta estrategia en las tareas de componentes mayormente cognitivos o mayormente motores, quizá deberían interpretar las acciones que forman parte de los deportes como un continuo cognitivo-motor, es decir, desde una perspectiva más ecológica (Sánchez y Lejeune, 1999). Probablemente, sería interesante realizar más estudios orientados a discernir las diferencias del empleo de la imaginería, teniendo en cuenta las tareas motoras o técnicas a ejecutar. De ese modo, se podría profundizar más en las diferencias existentes entre representaciones mentales de tan diversos gestos deportivos, como por ejemplo ocurre en el rendimiento de élite.

Siguiendo a Mora y Díaz Ocejo (2008), algunos de los efectos que parecen claros para los que se puede emplear esta técnica con deportistas incluirían:

- Mejorar los gestos técnicos deportivos, ya que la imaginería supone un complemento en el aprendizaje y adquisición de las acciones técnicotácticas en el deporte. 
- Controlar las respuestas fisiológicas y la activación.

- Mejorar la atención, puesto que el deportista atiende a lo que debe hacer durante la competición.

- Aumentar la autoconfianza, ya que el deportista se ve ejecutando cierta acción con éxito, reforzando así su confianza.

- Solución de problemas, ya que se puede imaginar el proceso de solución apropiada de un problema.

- Afrontamiento en situaciones de recuperación de lesiones deportivas.

Contrariamente, han de tenerse en consideración las sugerencias de Wann (1997), para quien si bien la mayoría de los trabajos realizados en materia de imaginería parecen haber tenido resultados favorables, serían destacables las siguientes limitaciones:

- La imaginería no puede sustituir en ningún caso al entrenamiento físico-técnico. Es decir, sobre todo en pruebas técnicas como un salto de altura en atletismo, el visualizar un salto no significa que alguien que nunca haya ejecutado esa acción sea capaz de lograrlo.

- La técnica parece ser menos efectiva en deportes donde predominan patrones motores gruesos, que en aquellas especialidades donde sí es necesario un alto componente técnico (cognitivo), como ocurre en el patinaje artístico sobre hielo, o los concursos en el atletismo. No obstante, esta afirmación ha de ser tenida en consideración como meramente orientativa, ya que, como apuntan Sánchez y Lejeune (1999), todos los deportes gozan, de algún modo, de componentes cognitivo y motores en sus acciones y gestos técnicos (componente cognitivo-motor).

- Imaginar un gesto técnico no es sinónimo de mejora de rendimiento deportivo. La imaginería positiva fomenta el rendimiento, sin embargo, la negativa puede deteriorarlo.

La Psicología del deporte ha sugerido algunos programas para fomentar el empleo del entrenamiento en imaginería. Algunos ejemplos de ello han sido el Visuo-Motor Behavior Rehearsal (VMBR) de Suinn (1972, 1976), el Sport Imagery Training (SIT) de Martens (1987) o el entrenamiento del programa de imaginería de Palmi (1987), todos con el objetivo de potenciar las posibilidades del empleo de esta estrategia cognitiva en el deporte de competición. Acerca de lo que nos espera en el futuro en esta parcela del entrenamiento mental, no sería sorprendente pensar, a raíz de las aportaciones en neurociencias de autores como Posner y Raichle (1994), que en un futuro próximo las investigaciones a través de la técnica PET (positron emision tomography) nos permita comprender mejor los mecanismos subyacentes en la representación en imaginería visual. 
Igualmente, y siguiendo algunas orientaciones contemporáneas, referidas a los efectos facilitadores, en contraposición a los debilitadores de esta estrategia, se abren nuevas posibilidades para una mejor compresión de cómo se comporta la imaginería.

Como hemos visto, se trata de una herramienta a través de la cual se incrementan las posibilidades de integrar las representaciones mentales de un modo más óptimo en el ámbito de la Actividad Física y el Deporte. Y estas parcelas del saber no son diferentes de la vida.

\section{REFERENCIAS}

ANNETT, J. (1988). "Imagery and skill acquisition". En M. Dennis, J. Engelkamp y J. T. E. Richardson (Eds.). Cognitive and neuropsysiological approaches to mental imagery (259-268). Dordrecht, The Netherlands: Martinus Nijhoff.

ANNETT, J. (1994). "The learning of motor skills: Sports science and ergonomics perspectives". Ergonomics, 37, 5-15.

ASHEN, A. (1984). ISM: "The Triple Code Model for imagery and psychophysiology". Journal of Mental Imagery, 8(4), 15-42.

BANDURA, A. (1977). Social learning theory. Englewood Cliffs, NJ: Prentice-Hall.

BRUNER, J. S. (1960). Toward a theory of instruction. Cambridge, Mass.: Harvard University Press.

BUDNEY, A. J., MURPHY, S. M. y WOOLFOLK, R. L. (1994). "Imagery and motor performance: What do we really know?". En A. A. Sheikh y E. R. Korn (Eds.), Imagery in sports and physical performance. (pp. 97-120). Amityville, NY: Baywood.

CALLOW, N. y HARDY, L. (2004). "The relationship between the use of kinaesthetic imagery and different visual imagery perspectives". Journal of Sports Sciences, 22, 167-177.

CARROLL, D., MARZILLIER, J. S., y MERIAN, S. (1982). "Psychophysiological changes accompanying different types of arousing and relaxing imagery". Psychophysiology, 19, 75-82.

CARROLL, S. J. y TOSI, H. L. (1973). Management by objectives: application and research. Macmillan, New York, NY.

CAUTELA, J. R. y SAMBPERIL, L. (1989). "Imagatletics: The applications of covert conditioning to athletic performance". Journal of Applied Sport Psychology, 1, 82-97.

CHIAIE, R., D’IPPOLITO, A., GRAMACCIONI, G., POLANI, D., REDA, M. A., RÉGINE, F., ROBAZZA, C. y SACCO, G. (2004). "El control 
de las emociones". En S. Tamorri (ed.), Neurociencias y deporte. Psicología deportiva. Procesos mentales del atleta (pp. 205-220). Barcelona: Paidotribo.

CORBIN, C. B. (1972). "Mental practice". En W.P. Morgan (Ed.), Ergogenic Aids and Muscular Performance (pp. 93-118). New York: Academic Press.

CUMMING, J., y STE-MARIE, D. M. (2001). "The cognitive and motivational effects of imagery training: A matter of perspective". The Sport Psychologist, 15, 276-287.

DENIS, M. (1985). "Visual imagery and the use of mental practice in the development of motor skills". Canadian Journal of Sports Science, 10, 45-165.

DÍAZ OCEJO, J. (2006). Análisis de las estrategias cognitivas en algunos deportes individuales y de adversario. Tesis Doctoral no publicada. Universidad de Málaga.

ECCLES, J. (1972). "Possible synaptic mechanisms subserving learning". En A. Karyman y J. Eccles (Eds.), Brain and human behavior. New York: Springer-Verlag.

EPSTEIN, M. L. (1980). "The relationships of mental imagery and mental practice to performance of a motor task". Journal of Sport Psychology, 2, 211-220.

GOSS, S., HALL, C. R., BUCKOLZ, E. y FISHBURNE, G. (1986). "Imagery ability and the acquisition and retention of movements". Memory and Cognition, 14, 469-477.

GOULD, D. y DARMARJIAN, N. (1996). "Imagery training for peak performance”. En J. L. Van Raalte y B. W. Brewer, Exploring Sport and Exercise Psychology (pp. 25-50). Washington, D. C.: APA.

HALE, B. D. (1982). "The effects of internal and external imagery on muscular and ocular concomitants". Journal of Sport Psychology, 4, 379-387.

HALE, B. D. (1994). "Imagery perspectives and learning in sports performance". En A. A. Sheikh y E. R. Korn (Eds.), Imagery in sports and physical performance (pp. 75-96). Amityville, N.Y: Baywood.

HALL, C. R. (2001). "Imagery in Sport ad Exercise". En R. N. Singer, H. A. Hasenblas y C. M. Janelle (Eds.). Handbook of Sport Psychology (2nd edition). (pp. 529-549). New York: John Wiley and Sons.

HALL, C. R. y PONGRAC, J. (1983). Movement Imagery Questionnaire. London, Canada: University of Western Ontario.

HALL, C. R., BUCKOLZ, E. y FISHBURNE, G. J. (1989). "Searching for a relationship between imagery ability and memory of movements". Journal of Human Movement Studies, 17, 89-100. 
HALL, C. R., MACK, D., PAIVIO, A. y HAUSENBLAS, H. (1998). "Imagery use by athletes: Development of the Sport Imagery Questionnaire". International Journal of Sport Psychology, 29, 73-89.

HALL, C. R., SCHMIDT, D., DURAND, M. y BUCKOLZ, E. (1994). "Imagery and motor skills acquisition". En A. A. Sheikh y E. R. Korn (Eds.). Imagery in sports and physical performance (pp. 121-134). Amityville, NY: Baywood.

HARDY, L. y CALLOW, N. (1999). "Efficacy of external and internal visual imagery perspectives for the enhancement of performance on tasks in which form is important". Journal of Sport and Exercise Psychology, 21, 95-112.

HARRIS, D. V. y ROBINSON, W. J. (1986). "The effects of skill level on EMG activity during internal and external imagery". Journal of Sport Psychology, 8, 105-111.

HERNÁNDEZ MENDO, A. (2002). "Imágenes mentales en el deporte”. En J. Dosil (Ed.), Psicología y rendimiento deportivo. (pp.83-102). Ourense: Gersam.

HOLMES, P. S. y COLLINS, D. J. (2001). "The PETTLEP Approach to Motor Imagery: A Functional Equivalence Model for Sport Psychologists". Journal of Applied Sports Psychology, 13 (1), 60-83.

JOHNSON, P. (1982). "The functional equivalence of imagery and movement". Quarterly Journal of Experimental Psychology, 34 (A), 349-365.

KOHL, R. M. y ROENKER, D. L. (1980). "Bilateral transfer as a function of mental imagery". Journal of Motor Behavior, 12, 197-206.

KOHL, R. M. y ROENKER, D. L. (1983). "Mechanism involvement during skill imagery, Journal of Motor Behavior, 15, 179-190.

LANG, P. J. (1977). "Imagery in therapy: An information processing analysis of fear". Behavior Therapy, 8, 862-886.

LANG, P. J. (1979). "A bio-information theory of emotional imagery". Psychophysiology, 16, 495-512.

LANG, P. J., MELAMED, B. G. y HART, J. A. (1970). “A psychophysiological analysis of fear modification using an automated desensibilization procedure". Journal of Abnormal Psychology, 76, 229-234.

MACKAY, D. G. (1981). "The problem of rehearsal or mental practice". Journal of Motor Behavior, 13, 274-285.

MAHONEY, M. J. y AVENER, M. (1977). "Psychology of the elite athlete: An exploratory study". Cognitive Therapy and Research, (1), 2, pp. 135141.

MARTENS, R. (1987). Coaches guide to sport psychology. Champaign, Illinois: Human Kinetics. 
McKAY, D. (1981). "The problem of rehearsal or mental practice". Journal of Motor Behavior, 13, 274-285.

McPHERSON, S. y VICKERS, J. N. (2004). "Cognitive control in motor expertise". International Journal of Sport and Exercise Psychology. 2, 274-300.

MEYERS, A. W., COOKE, C. J., CULLEN, J. y LILES, L. (1979). "Psychological aspects of athletic competitors: A replication across sports". Cognitive Therapy and Research, (3), 4, pp. 361-366.

MORA, J. A. y DÍAZ OCEJO, J. (2008). Control del Pensamiento y sus Estrategias en el Deporte. EOS: Madrid.

MORRISETT, L. N. (1956). The role of implicit practice in learning. Unpublised doctoral dissertation, Yale University, New Haven, CT.

MUMFORD, P. y HALL, C. (1985). "The effects of internal and external imagery on performing figures in figure skating". Canadian Journal of Applied Sport Sciences, 10, 171-177.

MURPHY, S. M. y JOWDY, P. (1992). "Imagery and Mental Practice". En T. S. Horn (Ed.), Advances in sport psychology, (pp. 221-250), Champaign, Ill.: Human Kinetics.

MURPHY, S. M. y MARTIN, K. A. (2002). "The use of imagery in sport". En S. T. Horn, (Ed); Advances in sport psychology (2nd ed.). pp. 405-439. Champaign, IL.: Human Kinetics.

NORDIN, S. M. y CUMMING, J. (2008). “Types and Functions of Athletes' Imagery: Testing Predictions from the Applied Model of Imagery Use by Examining Effectiveness". International Journal of Sport and Exercise Psychology, vol. 6, 2, pp. 189-206.

PAIVIO, A. (1985). "Cognitive and motivational functions of imagery in human performance". Canadian Journal of Applied Sport Sciences, 10, $22-28$.

PAIVIO, A. (1986). Mental representations: A dual coding approach. New York: Oxford University Press.

PALMI, J. (1987). "Práctica imaginada, l’entrenament ideomotor". Apunts Educació Física, 9, 26-31.

PERRY, H. M. (1939). "The relative efficiency of actual and imagery practice in five selected tasks". Archives of Psychogy, 34, 5-75.

PIAGET, J. y INHELDER, B. (1971) Mental Imagery in the Child. New York: Basic Books.

POSNER, M. I. y RAICHLE, M. E. (1994), Images of Mind, New York: Scientific American.

RAMSEY, R., CUMMING, J. y EDWARDS, M. G. (2008). "Exploring a Modified Conceptualization of Imagery Direction and Golf Putting Performance". International Journal of Sport and Exercise Psychology, vol. 6, 2, pp. 207-223. 
Estrategias representacionales en deportistas...

RANGANATHAN, V. K., SIEMIONOW, V., LIU, J. Z., SAHGAL, V. y YUE, G. H. (2004). "From mental power to muscle power-gaining strength by using the mind". Neuropsicología, 42, 944-956.

RAWLINGS, E. J., RAWLINGS, J. L., CHEN, S. S y YILK, M. D. (1972). “The facilitating effects of mental practice in the acquisition of rotary pursuit tracking. Psychonomic Science, 26, 71-73.

RYAN, E. D. y SIMONS, J. (1983). "What is learned in mental practice of motor skills? A test of the cognitive-motor hypothesis". Journal of Sport Psychology, 5, 419-426.

SACKETT, R. S. (1934). "The influences of symbolic rehearsal upon the retention of a maze habit". Journal of General Psychology, 10, 376-395.

SACKETT, R. S. (1935). "The relationship between amount of symbolic rehearsal and retention of a maze habit". Journal of General Psychology, $13,113-128$.

SAGE, G. (1977). Introduction to motor behavior: A neuro-psychological approach. Boston, MA: Addison-Wesley.

SÁNCHEZ, X. y LEJEUNE, M. (1999). "Práctica mental y deporte: ¿Qué sabemos después de casi un siglo de investigación?" Revista de Psicología del Deporte, 8, (1), pp. 21-37.

SCHACK, T. y TENENBAUM, G. (2004). "Effect Representation and Action Planning: A Preface". Journal of Sport and Exercise Psychology, 2, pp. 343-345.

SCHMIDT, R. A. (1982). Motor control and learning: A behavioral emphasis. Champaign, Ill.: Human Kinetics.

SHEIKH, A. y KUNZENDORF, R. (1984). "Imagery, physiology, and somatic illness". En A. Sheikh (Ed.), International Review of Mental Imagery. New York: Human Sciences.

SINGER, R. N. (1980). Motor learning and human performance. New York: Macmillan.

SMITH, D., WRIGHT, C., ALLSOPP, A. y WESTHEAD, H. (2007). "It's all in the mind: PETTLEP-based imagery and sports performance". Journal of Applied Sport Psychology, 19, 80-92.

SMYTH, M. M. (1975). "The role of mental practice in skill acquisition". Journal of Motor Behavior, 7, 199-206.

START, K. B. y RICHARDSON, A. (1964). "Imagery and mental practice". British Journal of Education Psychology, 34, 280-284.

SUINN, R. (1993). "Imagery" in Handbook of Research in Sport Psychology. En Singer, R. N., Murphey, M. y Tennant, L.K. (eds.) New York: Macmillan Publishing Co.

SUINN, R. M. (1976). "Body thinking: Psychology for Olympic champs". Psychology Today, pp. 38-43. 
SUINN, R. M. (1972). "Behavior rehearsal training for ski racers". Behavior Therapy, 3, 519-520.

VEALEY, R. S. (2007). "Mental Skills Training in Sport". En Tenenbaum, G. y Eklund, R. C. (Eds.) Handbook of Sport Psychology, $3^{\text {rd }}$ edition. Wiley, pp 287-309.

VEALEY, R. S. y WALTER, S. M. (1993). "Imagery training for performance enhancement and personal growth. En J. M. Williams (Ed.), Applied Sport Psychology: Personal Growth to peak performance. (pp. 200-224). Mountain View, CA: Mayfield.

WANN, D. L. (1997). Sport Psychology, Upple Saddle River, NJ: Prentice Hall.

WEINBERG, R. S. y GOULD, D. (2003). Foundations of Sport And Exercise Psychology. Champaign, Il: Human Kinetics.

WEINBERG, R. S., BUTT, J., KNIGHT, B., BURKE, K. L. y JACKSON, A. (2003). "The relationship between the use and effectiveness of imagery: An exploratory investigation”. Journal of Applied Sport Psychology, 15, 26-40.

WRISBERG, C. A. y RAGSDALE, M. R. (1979). "Cognitive demand and practice level: Factors in the mental practice of motor skills". Journal of Human Movement Studies, 5, 201-208. 